\title{
A meeting with Enrico Fermi
}

\section{How one intuitive physicist rescued a team from fruitless research.}

\section{Freeman Dyson}

$\mathrm{O}$ ne of the big turning points in my life was a meeting with Enrico Fermi in the spring of 1953. In a few minutes, Fermi politely but ruthlessly demolished a programme of research that my students and I had been pursuing for several years. He probably saved us from several more years of fruitless wandering along a road that was leading nowhere. I am eternally grateful to him for destroying our illusions and telling us the bitter truth.

Fermi was one of the great physicists of our time, outstanding both as a theorist and as an experimenter. $\mathrm{He}$ led the team that built the first nuclear reactor in Chicago in 1942. By 1953 he was head of the team that built the Chicago cyclotron, and was using it to explore the strong forces that hold nuclei together. He made the first accurate measurements of the scattering of mesons by protons, an experiment that gave the most direct evidence then available of the nature of the strong forces.

At that time I was a young professor of theoretical physics at Cornell University, responsible for directing the research of a small army of graduate students and postdocs. I had put them to work calculating meson-proton scattering, so that their theoretical calculations could be compared with Fermi's measurements. In 1948 and 1949 we had made similar calculations of atomic processes, using the theory of quantum electrodynamics, and found spectacular agreement between experiment and theory. Quantum electrodynamics is the theory of electrons and photons interacting through electromagnetic forces. Because the electromagnetic forces are weak, we could calculate the atomic processes precisely. By 1951, we had triumphantly finished the atomic calculations and were looking for fresh fields to conquer. We decided to use the same techniques of calculation to explore the strong nuclear forces. We began by calculating meson-proton scattering, using a theory of the strong forces known as pseudoscalar meson theory. By the spring of 1953, after heroic efforts, we had plotted theoretical graphs of meson-proton scattering.We joyfully observed that our calculated numbers agreed pretty well with Fermi's measured numbers. So I made an appointment to meet with Fermi and show him our results. Proudly, I rode the Greyhound bus from Ithaca to Chicago with

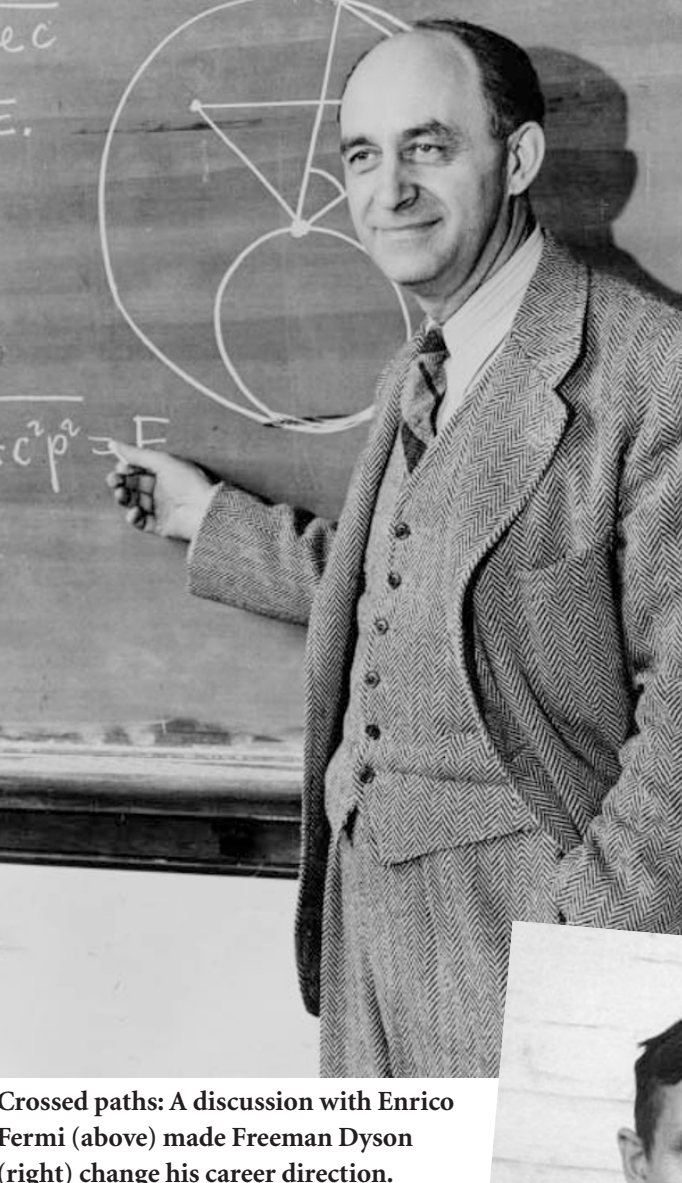

physical picture, and the forces are so strong that nothing converges. To reach your calculated results, you had to introduce arbitrary cut-off procedures that are not based either on solid physics or on solid mathematics."

In desperation I asked Fermi whether he was not impressed by the agreement between our calculated numbers and his measured numbers. He replied, "How many arbitrary parameters did you use for your calculations?" I thought for a moment about our cut-off procedures and said, "Four." He said, "I remember my friend Johnny von Neumann used to say, with four parameters I can fit an elephant, and with five I can make him wiggle his trunk." With that, the conversation was over. I thanked Fermi for his time and trouble, and sadly took the next bus back to Ithaca to tell the bad news to the students. Because it was important for the students to have their names on a published paper, we did not abandon our calculations immediately. We finished them and wrote a long paper that was duly published in the Physical Review with all our names on it. Then we dispersed to find other lines of work. I escaped to Berkeley, California, a package of our theoretical graphs to show to Fermi.

When I arrived in Fermi's office, I handed the graphs to Fermi, but he hardly glanced at them. He invited me to sit down, and asked me in a friendly way about the health of my wife and our newborn baby son, now fifty years old. Then he delivered his verdict in a quiet, even voice. "There are two ways of doing calculations in theoretical physics", he said. "One way, and this is the way I prefer, is to have a clear physical picture of the process that you are calculating. The other way is to have a precise and selfconsistent mathematical formalism. You have neither." I was slightly stunned, but ventured to ask him why he did not consider the pseudoscalar meson theory to be a selfconsistent mathematical formalism. $\mathrm{He}$ replied, "Quantum electrodynamics is a good theory because the forces are weak, and when the formalism is ambiguous we have a clear physical picture to guide us. With the pseudoscalar meson theory there is no to start a new career in condensed-matter physics.

Lookingback after fifty years, we can clearly see that Fermi was right. The crucial discovery that made sense of the strong forces was the quark. Mesons and protons are little bags of quarks. Before Murray GellMann discovered quarks, no theory of the strong forces could possibly have been adequate. Fermi knew nothing about quarks, and died before they were discovered. But somehow he knew that something essential was missing in the meson theories of the 1950s. His physical intuition told him that the pseudoscalar meson theory could not be right. And so it was Fermi's intuition, and not any discrepancy between theory and experiment, that saved me and my students from getting stuck in a blind alley.

Freeman Dyson is at the Institute for Advanced Study, Einstein Drive, Princeton, New Jersey 08540, USA. 